Article

\title{
Pathogenic Effect of GDAP1 Gene Mutations in a Yeast Model
}

\author{
Weronika Rzepnikowska ${ }^{1}$, Joanna Kaminska ${ }^{2} \mathbb{D}$, Dagmara Kabzińska ${ }^{1}$ and \\ Andrzej Kochański ${ }^{1, *}$ \\ 1 Neuromuscular Unit, Mossakowski Medical Research Centre Polish Academy of Sciences, 02-106 Warsaw, \\ Poland; wrzepnikowska@imdik.pan.pl (W.R.); dagkab@imdik.pan.pl (D.K.) \\ 2 Institute of Biochemistry and Biophysics Polish Academy of Sciences, 02-106 Warsaw, Poland; \\ kaminska@ibb.waw.pl \\ * Correspondence: akochanski@imdik.pan.pl; Tel.: +48-22-60-86-526
}

Received: 19 February 2020; Accepted: 12 March 2020; Published: 14 March 2020

check for updates

\begin{abstract}
The question of whether a newly identified sequence variant is truly a causative mutation is a central problem of modern clinical genetics. In the current era of massive sequencing, there is an urgent need to develop new tools for assessing the pathogenic effect of new sequence variants. In Charcot-Marie-Tooth disorders (CMT) with their extreme genetic heterogeneity and relatively homogenous clinical presentation, addressing the pathogenic effect of rare sequence variants within 80 CMT genes is extremely challenging. The presence of multiple rare sequence variants within a single CMT-affected patient makes selection for the strongest one, the truly causative mutation, a challenging issue. In the present study we propose a new yeast-based model to evaluate the pathogenic effect of rare sequence variants found within the one of the CMT-associated genes, GDAP1. In our approach, the wild-type and pathogenic variants of human GDAP1 gene were expressed in yeast. Then, a growth rate and mitochondrial morphology and function of GDAP1-expressing strains were studied. Also, the mutant GDAP1 proteins localization and functionality were assessed in yeast. We have shown, that GDAP1 was not only stably expressed but also functional in yeast cell, as it influenced morphology and function of mitochondria and altered the growth of a mutant yeast strain. What is more, the various GDAP1 pathogenic sequence variants caused the specific for them effect in the tests we performed. Thus, the proposed model is suitable for validating the pathogenic effect of known GDAP1 mutations and may be used for testing of unknown sequence variants found in CMT patients.
\end{abstract}

Keywords: yeast; mitochondria; Charcot-Marie-Tooth type 4 disease; GDAP1 gene; GDAP1 gene mutations

\section{Introduction}

In the past, molecular genetic testing was limited to single genes. Nowadays, whole exome sequencing (WES) is applied, the result of which is a series of DNA sequence variants even for the Mendelian diseases [1]. For example, in the hereditary peripheral neuropathies (Charcot-Marie-Tooth disorders; CMT) categorized as monogenic disorders (more than 80 genes involved), nearly $30 \%$ of the patients harbor two rare sequence and ten percent patients have three rare sequence variants in the CMT genes [2].

In Charcot-Marie-Tooth type 4A disease (CMT4A), causative mutations were identified in GDAP1 (ganglioside induced differentiation associated protein 1) [3,4]. The role of the GDAP1 gene in the physiology and pathology of the peripheral nerves is still not deciphered [5,6]. Recessive and dominant mutations within the GDAP1 gene segregate with severe and moderate to mild clinical progressions of the disease, respectively $[7,8]$. 
It appears that the pathogenic effect of the GDAP1 gene sequence variants reported until now varies substantially. However, different mutations were investigated in different manners. For some (causing Leu239Phe, Gly327Asp), the pathogenic effect has been documented by functional studies, whereas other characteristics are limited to clinical and electrophysiological observations. In addition, objective evaluation of the strength of any given mutation is hampered by the degree of mutation prevalence, which varies widely, i.e. some of the GDAP1 gene mutations were reported in numerous CMT families (Glu163X, Ser194X), whereas others (His123Arg, Glu222Lys) were reported in small and even single pedigrees [6].

Initially, the GDAP1 gene was identified as being induced upon enhanced ganglioside production [9]. Later it was shown that GDAP1 is a mitochondrial membrane protein mainly present in neuronal cells [10-12]. It is involved in mitochondria fission [13,14], calcium homeostasis [15,16], and maintenance of cellular redox potential $[17,18]$, although the exact function of GDAP1 in these processes is unknown.

In yeast there is no simple homolog of human GDAP1 gene, however it was shown that it complements some FIS1 gene deletion phenotypes, coding for protein involved in mitochondrial fission process [19]. These results suggest that GDAP1 is functional in yeast cells and possesses molecular partners.

The aim of this study was to develop a reproducible yeast-based system for determination of the pathogenicity of GDAP1 sequence variants. This system is based on the mitochondrial localization of GDAP1 protein and its mutations influence on mitochondria morphology and calcium signaling in mammalian cells. Here, we studied a localization of mutant GDAP1 proteins in yeast cells, its impact on mitochondrial network formation, the rate of mitochondrial DNA escape to the nucleus and the ability to grow on a non-fermentable carbon source. Additionally, we found that expression of wild-type GDAP1 gene reduces the growth defects of the yeast $\operatorname{csg} 2 \triangle$ mutant, with the deletion of a gene required for mannosylation of inositolphosphorylceramide, while mutated versions of GDAP1 do not. In summary, our model may help to identify potentially pathogenic GDAP1 alleles in CMT patients.

\section{Materials and Methods}

\subsection{Strains, Media, and Growth Conditions}

Escherichia coli strains DH5 $\alpha$ and XL1-Blue were used for plasmid propagation. The yeast Saccharomyces cerevisiae strains used in this study were BY4741 MATa his $3 \Delta 1$ leu2 $\Delta 0$ met $15 \Delta 0$ ura3 $\Delta 0$, BY4741 csg2 $\Delta$ and PTY44 MAT $\alpha$ ura3-52 lys2 leu2-3,112 trp1 1 [ [rho+, TRP1] [20].

Yeast were grown at $28{ }^{\circ} \mathrm{C}$ or $30^{\circ} \mathrm{C}$ in YPD medium ( $1 \%$ yeast extract, $2 \%$ peptone, $2 \%$ glucose), YPG medium (1\% yeast extract, $2 \%$ peptone, $3 \%$ glycerol) or YPG $+0.2 \%$ glucose, in minimal synthetic medium with glycerol $(0.67 \%$ yeast nitrogen base with ammonium sulfate without amino acids, $3 \%$ glycerol with desired supplements (uracil, amino acids)) or in complete synthetic medium (SC) $(0.67 \%$ yeast nitrogen base with ammonium sulfate without amino acids, $2 \%$ glucose or $3 \%$ glycerol with complete supplement mixture (CSM-ade-his-leu-trp-ura)) either solid or liquid. For growth tests, mitochondrial observations and mitochondrial DNA escape assays, a mixture of several yeast colonies, obtained after transformation, were grown overnight in liquid media. For growth tests the optical cell density $\left(\mathrm{OD}_{600}\right)$ was determined and cultures were diluted with water to $\mathrm{OD}_{600} \sim 1$. Subsequently, aliquots of 10 -fold serial dilutions of cells were spotted on solid media plates (as indicated) supplemented as indicated. Plates were incubated at $28^{\circ} \mathrm{C}$ for the indicated number of days.

\subsection{Plasmids}

The plasmids used in this study are listed in Table 1. 
Table 1. Plasmid used in this study.

\begin{tabular}{cc}
\hline Plasmid & Source or Reference \\
\hline $\mathrm{p}$ CMV6-XL5-GDAP1 & OriGene \\
$\mathrm{p} 425-\mathrm{P}_{T D H 3}[2 \mu ; L E U 2]$ & {$[21]$} \\
$\mathrm{p} 425-\mathrm{P}_{T D H 3}-G D A P 1$ & This study \\
$\mathrm{p} 425-\mathrm{P}_{T D H 3}-G D A P 1 m 1$ & This study \\
$\mathrm{p} 425-\mathrm{P}_{T D H 3}-G D A P 1 m 2$ & This study \\
$\mathrm{p} 425-\mathrm{P}_{T D H 3}-G D A P 1 m 3$ & This study \\
$\mathrm{p} 425-\mathrm{P}_{T D H 3}-G D A P 1 m 4$ & This study \\
$\mathrm{p} 425-\mathrm{P}_{T D H 3}-G D A P 1 m 5$ & This study \\
$\mathrm{p} 425-\mathrm{P}_{T D H 3}-G D A P 1 m 6$ & This study \\
$\mathrm{p} 425-\mathrm{P}_{T D H 3}-G D A P 1 m 7$ & This study \\
$\mathrm{p} 425-\mathrm{P}_{T D H 3}-G D A P 1 m 8$ & This study \\
PXY122 $(\mathrm{mt}-\mathrm{GFP})[2 \mu, H I S 3]$ & {$[22]$} \\
\hline
\end{tabular}

The $\mathrm{p} 425-\mathrm{P}_{T D H 3}-G D A P 1$ and $\mathrm{p} 425-\mathrm{P}_{T D H 3}-G D A P 1 m 1$ plasmids were produced by amplification of full length or truncated GDAP1 cDNA by PCR using a commercially available pCMV6-XL5 vector containing cDNA of GDAP1 (NM_018972; OriGene) as a template and primers providing restriction sites (BamHI at 5'-end and SalI 3'-end) and additional codon stop for GDAP1 $\mathrm{m} 1$ and ligation obtained alleles into p425-P $\mathrm{P}_{\mathrm{TDH} 3}$ vector. The $\mathrm{m} 2$ (c.980G >A; p.Gly327Asp), m3 (c.652C $>\mathrm{G}$; p.Gln218Glu), m4 (c.664G >A; p.Glu222Lys), m5 (c.715C> T; p.Leu239Phe), m6 (c.368A >G; p.His123Arg), m7 (c.347T>C; p.Met116Thr), and $\mathrm{m} 8$ (c.467C $>$ G; p.Ala156Gly) mutations were introduced by site directed mutagenesis into cDNA GDAP1 gene.

\subsection{Site Directed Mutagenesis}

Site directed mutagenesis was performed on the OriGene plasmid: pCMV6-XL5 with GDAP1 (NM_018972) Human Untagged Clone, using the Mut Express II Fast Mutagenesis Kit V2 (Vazyme) in accordance with the manufacturer's instructions.

Primers for mutagenesis were designed using Quick Change Primer Design (Agilent) on-line software (the primer sequences were placed in Table A1).

Escherichia coli strain XL1-Blue was used for plasmid propagation. The presence of mutations within constructed plasmids was verified by the Sanger sequencing method.

\subsection{Fluorescence Microscopy}

Mitochondria morphology was observed in yeast cells grown overnight in SC-leu-his media at $30{ }^{\circ} \mathrm{C}$ and transferred into SC gly-leu-his for $4 \mathrm{~h}$. The cells were viewed with an Eclipse E800 (Nikon, Tokyo, Japan) fluorescence microscope equipped with a DS-5Mc camera (Nikon). Images were collected using Lucia General 5.1 software (Laboratory Imaging Ltd., Prague, Czech Republic). The same fields were viewed by differential interference contrast (DIC) optics.

\subsection{Confocal Microscopy}

To observe the localization of GDAP1, yeast cells were grown overnight in SC-leu-his media at $28{ }^{\circ} \mathrm{C}$ and shifted into SC gly-leu-his for $4 \mathrm{~h}$. Cells were fixed by incubation for $25 \mathrm{~min}$ in $3.7 \%$ formaldehyde. They were collected by centrifugation and washed four times in buffer B $(40 \mathrm{mM}$ potassium phosphate, $\mathrm{pH} 7.0 ; 0.5 \mathrm{mM} \mathrm{MgCl} 2 ; 1.2 \mathrm{M}$ sorbitol). Then they were resuspended in buffer B supplemented with $0.2 \mathrm{mg} / \mathrm{mL}$ Zymolyase 20T (Amsbio, Abingdon, UK) and 1\% 2-mercaptoethanol and incubated for $1 \mathrm{~h}$ at $37^{\circ} \mathrm{C}$. The resulting spheroplasts were washed twice in buffer B and were spotted onto polylysine-coated slides. They were permeabilized by incubation in buffer $\mathrm{F}(20 \mathrm{mM}$ potassium phosphate, $\mathrm{pH} 7.4 ; 150 \mathrm{mM} \mathrm{NaCl} ; 0.1 \% \mathrm{BSA}$ ) supplemented with $0.1 \%$ Triton-X-100 for $15 \mathrm{~min}$. The slides were rinse with buffer $\mathrm{F}$ and saturated in buffer $\mathrm{F}$ for $1 \mathrm{~h}$. Then they were incubated with rabbit polyclonal anti-GDAP1 antibody (Abcam, Cambridge, MA, USA) diluted 1:200 in buffer F 
for $2 \mathrm{~h}$. They were washed with buffer $\mathrm{F}$ and incubated with TRITC-conjugated porc anti-rabbit IgG antibody (DAKO, Agilent, Santa Clara, CA, USA) diluted 1:60 for $2 \mathrm{~h}$. Then slides were rinsed with buffer F and nucleus was stained by DAPI (Thermo Fisher Scientific, Waltham, MA, USA) for 2 min. After being washed with water, slides were mounted in mounting medium (DAKO). Samples were viewed using LSM 780 Axio Observer Z.1 confocal microscope (Zeiss, Oberkochen, Germany). Images were collected using Zen 2012 black edition software (Zeiss). The confocal microscopy observations were performed in Laboratory of Advanced Microscopy Techniques, Mossakowski Medical Research Centre, PAS.

\subsection{Western Blot Analysis}

Yeast cells were grown overnight at $28^{\circ} \mathrm{C}$ in SC -leu medium. Protein extracts were prepared after disrupting cells with acid-washed glass beads in $2 \times$ urea electrophoresis sample buffer (50 mM Tris-HCl pH 6.8; 1.6\% SDS; 7\% glycerol; 0.016\% bromophenol blue; $4 \% \beta$-mercaptoethanol; $8 \mathrm{M}$ urea) supplemented with protease inhibitor cocktail (Sigma-Aldrich, Saint Louis, MI, USA). Samples were analyzed by standard Western blotting using rabbit polyclonal anti-GDAP1 (Abcam) or rabbit polyclonal anti-Histone H3 (Abcam) antibodies and secondary anti-rabbit IgG horseradish peroxidase (HRP)-conjugated antibodies (Sigma-Aldrich), followed by enhanced chemiluminescence (GE Healthcare, Boston, MA, USA).

\subsection{DNA Escape Assay}

DNA escape from mitochondria to the nucleus was assayed as in [20]. Briefly, the PTY44 strain was transformed with empty vector or one of the vectors bearing cDNA of GDAP1 variants. The transformants were grown in minimal synthetic medium with glycerol-leu overnight and aliquots were spread on SC-trp or diluted and spread on YPD to count colony forming units. The percentage of cells able to grow on SC-trp was calculated for each type of transformants.

\subsection{Estimation of Yeast Respiratory-Deficiency}

Cells were grown overnight in YPG medium, diluted, and spread on a medium containing glycerol (non-fermentable) as the main source of carbon and with a limited amount of fermentable glucose $(0.2 \%)$. We counted the fraction of small colonies (the respiratory deficient) whose growth was limited due to reduced levels of available fermentable glucose in relation to the total number of colonies.

\section{Results}

\subsection{Selection of GDAP1 Mutations}

From over 100 GDAP1 gene sequence variants, we have selected the eight most representative for CMT4A disease in the Polish families diagnosed and observed for at least 5 years in the Warsaw Neuromuscular Unit (clinical electrophysiological and morphological studies have been previously reported). The main selection criterion was our detailed knowledge of the phenotypes associated with each mutation that have been extensively characterized in our laboratory [23-26]. We refer to our selected mutations as GDAP1 $\mathrm{m} 1$ to $\mathrm{m} 8$ (Figure 1). Three mutations cause amino acid substitutions: Gly327Asp (m2), Leu239Phe (m5), and Met116Thr (m7), and belong to the class of mutations inherited as an autosomal recessive trait. The mutations Gly218Glu (m3), Ala156Gly (m8), and His123Arg (m6) represent pure dominant GDAP1 gene mutations. It was shown that the GDAP1 m4 mutation (Glu222Lys) is inherited in both dominant and recessive ways. The last mutation, that we called GDAP1 $\mathrm{m} 1$, results in a C-terminal truncation of the GDAP1 protein (to 287 amino acid residues). This mutation is representative for the two recurrent nonsense GDAP1 gene mutations i.e. Glu163X and Ser194X (Figure 1). 


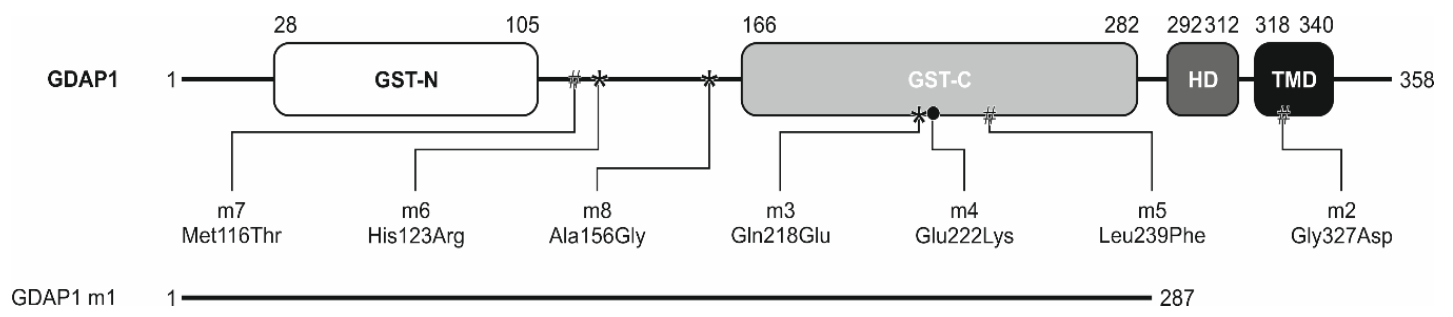

Figure 1. Schematic representation of the GDAP1 domain structure. Studied protein changes are indicated. GDAP1 $\mathrm{m} 1$ is a truncated version of GDAP1, lacking the domains responsible for correct localization of the protein (HD and TMD). Dominant mutations leading to the given substitution are flagged by stars $\left(^{*}\right)$ and recessive instances by a hashtag (\#). GDAP1 $\mathrm{m} 4$, which may be inherited in both dominant and recessive ways, is indicated by dot (•). Domains (based on Pfam database): (GST) glutathione S-transferase domain; (HD) hydrophobic domain; (TMD) transmembrane domain.

3.2. cDNA of the Human GDAP1 Gene Coding for Ganglioside Induced Differentiation Associated Protein 1 Is Stable When Heterologously Expressed in Yeast Cells

Due to the absence of homology between human GDAP1 and any yeast gene we were unable to apply the strategy usually used in complementation tests. To address whether we can use yeast cells to assess the severity of the pathogenicity of GDAP1 variants, we expressed the cDNA of the human wild-type GDAP1 gene, and of the GDAP1 variants, in yeast cells. First, we investigated the effect of GDAP1 cDNA expression on growth of the wild-type yeast cells. Expression of neither the wild-type GDAP1 nor any of the mutated variants (GDAP1 $\mathrm{m} 1-\mathrm{m} 8)$ affected the growth of yeast cultures on rich YPD (Figure 2A). To ensure that the GDAP1 variants are properly expressed, the total cell extracts obtained from cells transformed with empty vector or bearing cDNA of GDAP1 variants were prepared, separated by SDS-PAGE and immunoblotted with anti-GDAP1 antibodies. As a loading control, the levels of Histone 3 were monitored. Bands corresponding to GDAP1 and GDAP1 m1 proteins of appropriate molecular weights were observed (Figure 2B). The cDNAs of the missense GDAP1 gene variants (GDAP1 $\mathrm{m} 1, \mathrm{~m} 3, \mathrm{~m} 4, \mathrm{~m} 5, \mathrm{~m} 6, \mathrm{~m} 7, \mathrm{~m} 8)$ were also stably expressed in wild-type yeast cells (Figure 2B). Thus, we were able to prove that in a heterologous system, human GDAP1 variants are expressed and that their expression does not disturb the growth of wild-type yeast cell cultures. Due to the lack of any noticeable difference in growth between transformants expressing the cDNA of wild-type GDAP1 or of its mutants, we had to search for conditions that allowed us to differentiate between cells producing wild-type GDAP1 and cells producing mutant GDAP1 proteins.

\subsection{Specific GDAP1 Mutations Cause Mislocalization of the Resulting GDAP1 Protein}

GDAP1 protein was been characterized as being localized to the mitochondrial membrane in neuronal cells [10-12]. Thus, in order to further characterize human GDAP1 functionality in the heterologous yeast-based system, we analyzed its cellular localization by confocal microscopy. The yeast cells were transformed with a plasmid encoding a mitochondrially targeted green fluorescence protein (mt-GFP) [22], empty vector or a series of GDAP1 variants either with or without mutations. The wild-type GDAP1 protein was located in structures that matched the location of mt-GFP. Similarly, $\mathrm{m} 3, \mathrm{~m} 4, \mathrm{~m} 5, \mathrm{~m} 6$ and $\mathrm{m} 7$ mutations did not result in changes in the subcellular localization of these GDAP1 protein variants. Two mutations, namely $\mathrm{m} 1$ and $\mathrm{m} 2$ (causing a C-terminal deletion and the Gly327Asp substitution, respectively), resulted in GDAP1 proteins that did not localize to mitochondria (Figures 2C and A1). However, the level of fluorescence signal from the GDAP1 m2 protein was only slightly higher than the background signal observed for the empty vector control making it very difficult to observe the protein. The mislocalization of the GDAP1 $\mathrm{m} 1$ and $\mathrm{m} 2$ protein variants is most probably the result of an absence (in the case of GDAP1 m1) or functional impairment (for GDAP1 $\mathrm{m} 2$ ) of the transmembrane domain (TMD), which has previously been shown to be responsible for the mitochondrial membrane anchoring of GDAP1. Similarly to previous observations made in COS7 cells, 
mutations that resulted in changes within the TMD (e.g. GDAP1 m2) of the GDAP1 protein impaired the targeting of GDAP1 to the mitochondrial membrane [25].

A
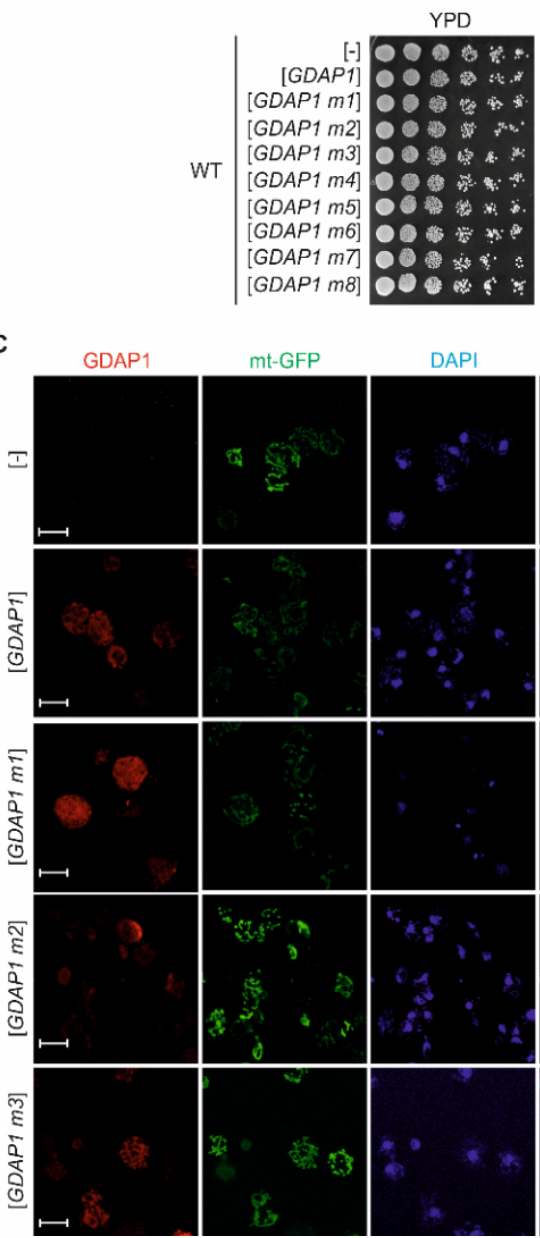

B

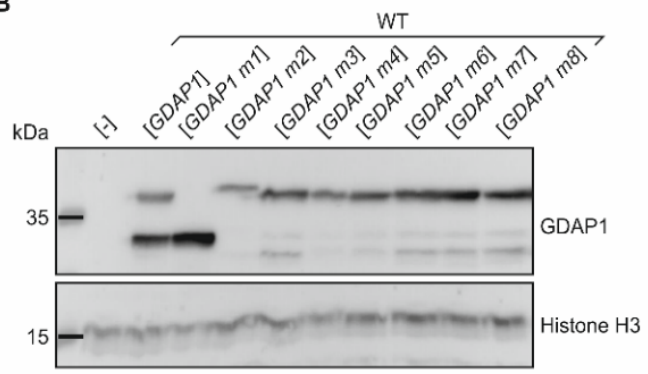

Merge

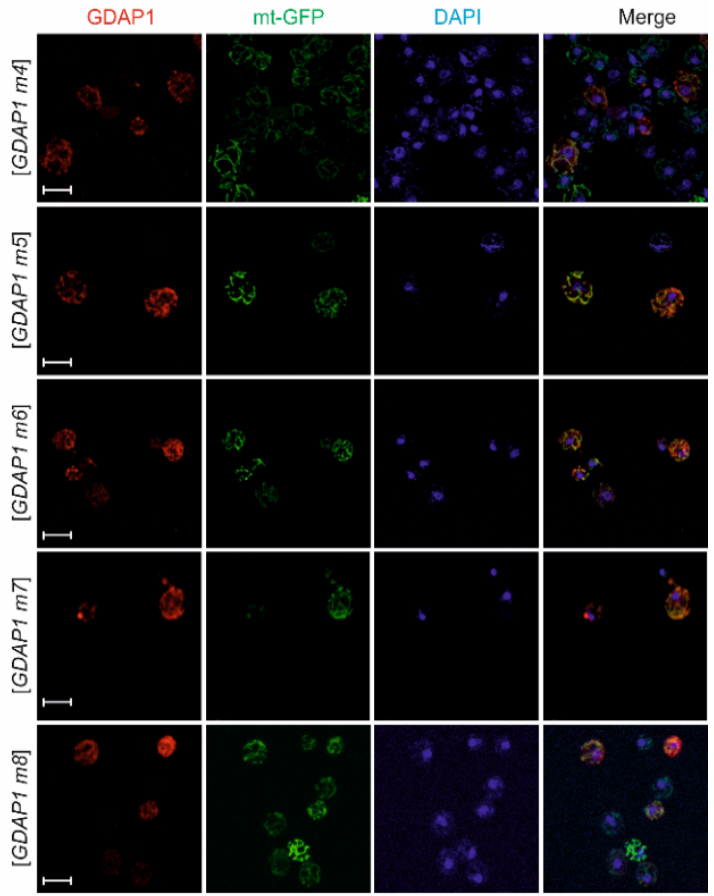

Figure 2. Yeast-based system to analyze GDAP1 protein function. (A) Overnight cultures of wild-type yeast cells harboring empty vector ([-]) or plasmids with GDAP1 gene variants were diluted to $\mathrm{OD}_{600}$ $\approx 1$ and then ten-fold serial dilutions were prepared and spotted on to YPD medium. Plates were incubated for $2 \mathrm{~d}$ at $28^{\circ} \mathrm{C}$. (B) Yeast wild-type cells harboring empty vector ([-]) or plasmids with GDAP1 gene variants (as indicated) were cultured overnight in SC-leu at $28^{\circ} \mathrm{C}$, then were collected and disrupted. The total cell extracts obtained were analyzed by SDS-PAGE, followed by immunoblotting with anti-GDAP1 or anti-Histone H3 antibodies, as indicated. (C) Yeast wild-type cells transformed with plasmid encoding mitochondrially targeted green fluorescent protein (mt-GFP) and with empty vector ([-]) or plasmids encoding GDAP1 alleles (as indicated) were grown in SC-leu-his medium overnight and then were shifted to glycerol-containing medium for $4 \mathrm{~h}$ to induce expansion of mitochondria. Cells were fixed, stained and observed using confocal microscopy. GDAP1 was visualized by immunostaining (red), mitochondria using the mt-GFP protein marker (green) and the nucleus was stained with DAPI (blue). Scale bar $5 \mu \mathrm{m}$.

\subsection{Expression of Human GDAP1 cDNA Affects Mitochondrial Network Formation in Yeast}

In previous studies, GDAP1 has been shown to alter the formation of mitochondrial networks when expressed in HeLa cells [23]. In our study we investigated whether expression of GDAP1 in the heterologous system also changes the morphology of mitochondrial networks, especially given that the human protein is also localized to the mitochondria. The mitochondria were visualized by expression of mt-GFP. The network of mitochondria of cells transformed with empty vector were used 
as a reference. As expected, mitochondria formed tubular network structures below the cell cortex. After the introduction of cDNA encoding human GDAP1 protein, we observed an alteration of the mitochondrial network. We named this a supernetwork due to the more extensive and branched nature of the network (Figure 3A). Compared to the wild-type yeast, about $50 \%$ of the counted GDAP1 transformed cells displayed this supernetwork phenotype. We also observed spherical and abnormal phenotypes, but there were no marked differences in the number of cells with such mitochondria between the cells expressing the cDNA of wild-type GDAP1 and those that were not (Figure 3A).

A

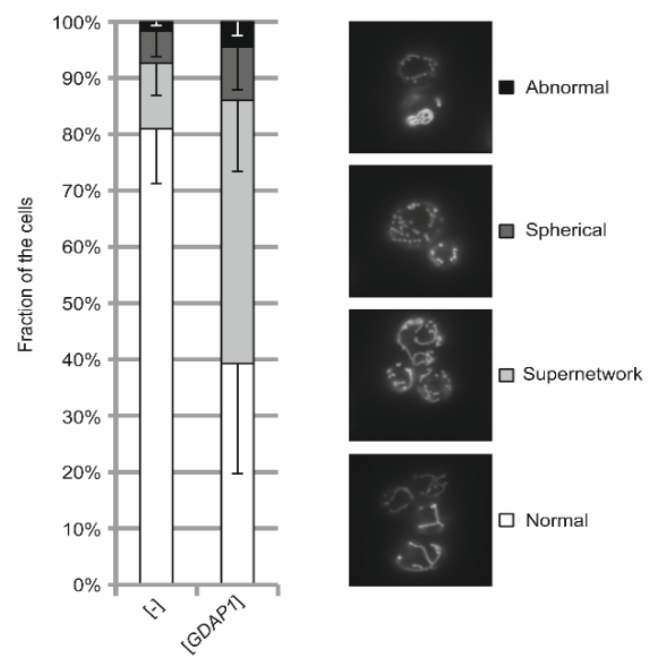

B

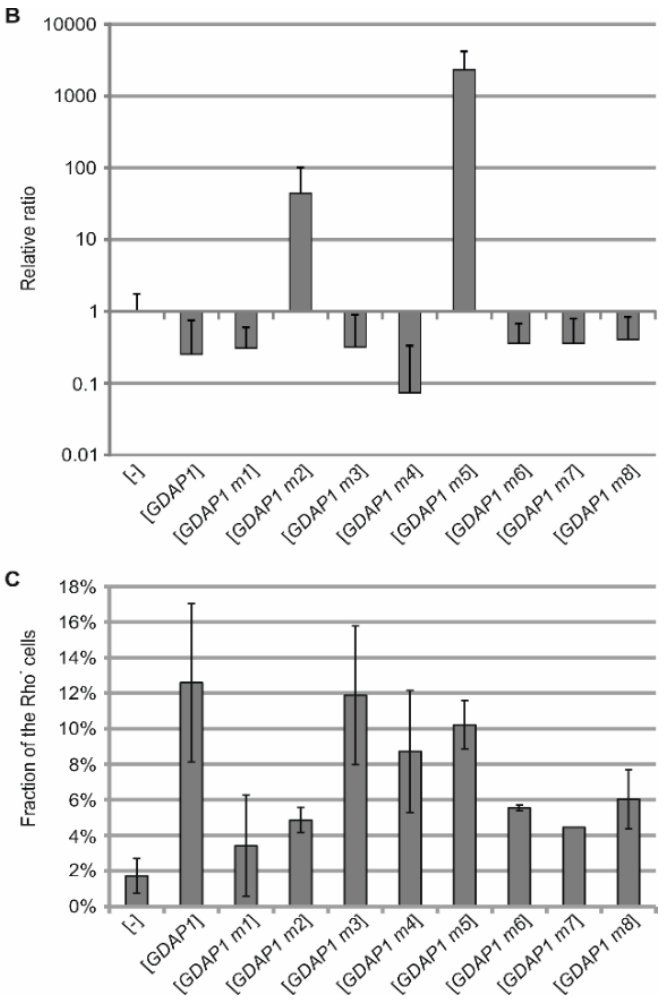

Figure 3. Mitochondria functioning is changed upon GDAP1 expression in yeast. (A) Overnight cultures of wild-type yeast cells harboring plasmid bearing mitochondrially targeted green fluorescent protein (mt-GFP) and empty vector ([-]) or plasmid with full-length GDAP1 were shifted to glycerol-containing medium for $4 \mathrm{~h}$ to induce expansion of mitochondria. Mitochondrial morphology was observed using fluorescent microscopy. The described phenotypes were counted. Error bars represent standard deviation for three independent biological experiments. (B) PTY44 strain transformed with empty vector ([-]) or GDAP1 gene variants (as indicated) was incubated for 2 days in glycerol-containing medium and then the cells were counted, and dilutions of every culture were plated on YPD or SC-trp medium. After $3 \mathrm{~d}$ of incubation at $28^{\circ} \mathrm{C}$ the number of colony-forming units (CFUs) was calculated. The fraction of SC-trp-growing cells in every culture were normalized to PTY44 with empty vector ([-]). Error bars represent standard deviation for four experiments. (C) Wild-type yeast strain harboring empty vectors ([-]) or plasmids with GDAP1 alleles were grown overnight in SC gly-leu medium. The cultures were diluted, and cells were plated on YPG $+0.2 \%$ glucose and incubated for $3 \mathrm{~d}$ at $28^{\circ} \mathrm{C}$. $\mathrm{CFUs}$ were counted and the percentage of small colonies was calculated. Error bars represent standard deviation for four experiments.

We have confirmed the role of human GDAP1 protein in the formation of the mitochondrial network in yeast cells. The phenotypes we observed, however, were not suitable to distinguish between GDAP1 mutants or to use as a prognostic marker to assess pathogenicity of GDAP1 mutations. 


\subsection{Some GDAP1 Protein Mutants When Produced in Yeast Increase the Rate of Mitochondrial DNA Escape to the Nucleus}

In the previous experiment we have shown that expression of the cDNA from the human GDAP1 gene influences the formation of the mitochondrial network in yeast. Moreover, a relationship between mitochondrial network disturbances and mutations in the GDAP1 gene was reported $[12,14]$. Taking into account that the mitochondrial network formation is directly associated with mitochondrial biogenesis and that this process is accompanied by an exchange of mitochondrial DNA $[27,28]$, we decided to check the functionality of mitochondria by assessment of mtDNA escape to the nucleus [29]. Thus, we hypothesized that at least some GDAP1 gene mutations may affect mitochondrial DNA escape; from the mitochondria to the nucleus. To measure the rate of mtDNA escape to the nucleus, the TRP1 gene, encoding an enzyme from the tryptophan biosynthesis pathway, was integrated into the mitochondrial genome $[20,30]$ in a strain carrying a defective trp 1 allele in the (nuclear) genome. Such yeast is unable to grow without tryptophan supplementation. During the propagation of such yeast, mtDNA escapes from the mitochondria to the nucleus where it integrates and complements the defective nuclear TRP1 allele allowing cells to grow on the media without tryptophan [20,31].

The idea of this experiment may be summarized by the following simple association: more extensive abnormal mitochondria leads to a greater rate of DNA escape and reflects the severity of the GDAP1 gene mutation. The greatest mitochondrial DNA escape rate was observed when the cDNA of GDAP1 $\mathrm{m} 5$ (resulting in the Leu239Phe substitution) was expressed (Figure 3B). A moderate effect was observed for GDAP1 m2 (Gly327Asp substitution within the TMD). The effect of expressing other GDAP1 variants was not strong. There were no statistically relevant differences in mtDNA escape rates between cells bearing empty plasmid or cDNA of the GDAP1 $\mathrm{m} 1, \mathrm{~m} 3, \mathrm{~m} 4, \mathrm{~m} 6, \mathrm{~m} 7$, and $\mathrm{m} 8$ variants. Altogether, these results show that although the expression of GDAP1 does not affect the growth of yeast cells, it does affect their physiology, in this instance the mitochondria. The changes are so extensive that they can be observed. This supports the idea of using a yeast model to study pathologies caused by mutations in the GDAP1 gene.

An altered morphology of mitochondria is often associated with a defective respiratory function [32]. We asked whether the observed changes in the morphology of mitochondria translate into the ability to grow on a non-fermentable carbon source, the conditions requiring functional mitochondrial respiration. The expression of wild type GDAP1 caused a significant increase in the number of cells which are unable to grow on non-fermentable carbon source containing media $(p<0.05)$ and thus are respiratory deficient (Figure $3 C$ ). This effect was significantly reduced when GDAP1 $\mathrm{m} 1, \mathrm{~m} 2, \mathrm{~m} 6$, and $\mathrm{m} 7$ variants were expressed $(p<0,05)$ and was not altered by GDAP1 m3, m4, and $\mathrm{m} 5$ variants (Figure $3 \mathrm{C}$ ). The decrease in the number of respiratory deficient cells between yeast transformants expressing different pathogenic GDAP1 variants and the wild type GDAP1 reflects the influence of mutations on GDAP1 protein function connected with the observed phenotype.

\subsection{The Expression of Human Wild-Type GDAP1 Gene Reduces the Growth Defect of the csg2 Mutant Yeast Strain}

The yeast-based model of the CMT4A disease that is obtained through the heterologous expression of the cDNA of GDAP1 and its eight mutants represents a stable and functional system which has several limitations. The main limitation lies in the mutation-specific phenotype. In essence, only some GDAP1 mutations may be analyzed using the mtDNA escape assay. Similarly, the phenotypes based upon colocalization of the GDAP1 with mitochondria are only suitable to distinguish whether mutations affect the transmembrane domain of GDAP1. To obtain a simple model to use as a potential platform for further drug screening, we looked for a repeatable growth phenotype.

Based on GDAP1 localization data obtained from the literature, on the abnormalities described as being associated with GDAP1 mutations and on data from studies of other genes in which mutations also cause CMT disease, we expressed the cDNA of GDAP1 in several specifically selected yeast deletion mutants. We assumed that we should be able to find a mutant and/or conditions which 
allow us to monitor GDAP1 expression in simple growth test. One of our candidates was $\operatorname{csg} 2 \Delta$ mutant, with the deletion of a gene required for mannosylation of inositolphosphorylceramide. This mutant has higher levels of reactive oxygen species (ROS) and loss of mitochondrial DNA suppress this accumulation [33]. Thus, we suspected that expression of GDAP1 or its variants which results accumulation of Rho ${ }^{-}$cells could be helpful for growth of $\operatorname{csg} 2 \Delta$ at stress conditions. We found that the tunicamycin and calcium ion $\left(\mathrm{Ca}^{2+}\right)$ sensitivity of the $\operatorname{csg} 2 \Delta$ strain is suppressed by expression of wild-type the GDAP1 allele (Figure 4A). The truncated version of GDAP1 protein (GDAP1 m1) and the mutation in which localization to the mitochondria is perturbed (GDAP1 m2) were not able to restore the normal growth of mutant cells on either plates containing tunicamycin or $\mathrm{Ca}^{2+}$ ions. Similar to GDAP1 $\mathrm{m} 1$, the expression of GDAP1 $\mathrm{m} 3$ was unable to restore growth of the $\operatorname{csg} 2 \Delta$ cells in either test condition. The expression of GDAP1 $\mathrm{m} 5, \mathrm{~m} 6$, and $\mathrm{m} 7$ restored the growth of the $\operatorname{csg} 2 \Delta$ mutant to a lesser degree; weaker when compared to the wild-type GDAP1 but stronger than GDAP1 $\mathrm{m} 1$. In these terms the mutations GDAP1 $\mathrm{m} 1, \mathrm{~m} 2, \mathrm{~m} 3, \mathrm{~m} 5, \mathrm{~m} 6, \mathrm{~m} 7$, and $\mathrm{m} 8$ could be classified as loss-of-function or partial loss-of-function mutations. Interestingly in this assay the effect of the Glu222Lys substitution caused by the GDAP1 $\mathrm{m} 4$ mutation was negligible and the growth of the $\operatorname{csg} 2 \triangle$ mutant expressing GDAP1 $\mathrm{m} 4$ was fully restored on tunicamycin and restored to a slightly lesser extent on $\mathrm{Ca}^{2+}$ ion containing medium, in comparison to wild-type GDAP1 expression (Figure 4A). The lack of complementation by some GDAP1 variants is not due to lack of protein expression as shown by Western blot analysis of GDAP1 proteins (Figure 4B). In summary, our study has identified a new phenotype connected with expression of the GDAP1 cDNA in yeast cells. This supported our hypothesis that we should be able to identify conditions under which the presence of GDAP1 protein can be studied in a simple growth assay. This test may help to identify at least some of the loss-of-function alleles found in patients.

A

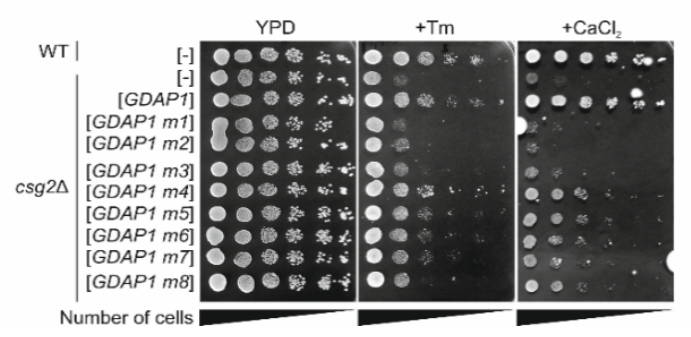

B

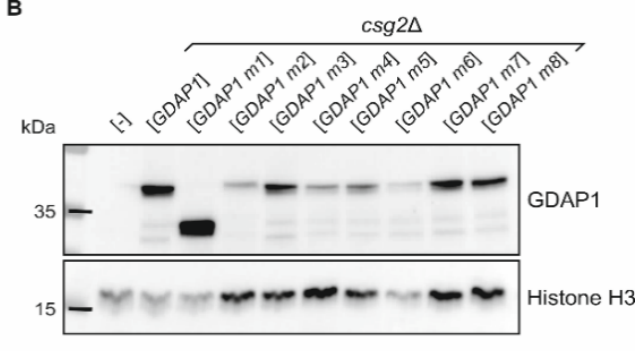

Figure 4. Possible yeast-based model of CMT4A disease for drug screen. (A) Overnight cultures of wild-type or $\operatorname{csg} 2 \triangle$ yeast cells harboring empty vector ([-]) or plasmids with GDAP1 alleles were diluted to $\mathrm{OD} 600 \approx 1$. Ten-fold serial dilutions were prepared and spotted on YPD media containing $0.5 \mu \mathrm{g} / \mathrm{mL}$ tunicamycin $(\mathrm{Tm})$ or $0.5 \mathrm{M}$ calcium chloride $\left(\mathrm{CaCl}_{2}\right)$. Plates were incubated for $2 \mathrm{~d}$ (YPD and $\left.+\mathrm{Tm}\right)$ or $6 \mathrm{~d}\left(+\mathrm{CaCl}_{2}\right)$ at $28^{\circ} \mathrm{C}$. (B) The $\operatorname{csg} 2 \Delta$ yeast strain harboring empty vector ([-]) or plasmids with GDAP1 alleles (as indicated) were cultured overnight in SC-leu medium at $28^{\circ} \mathrm{C}$. Cells were then harvested and disrupted. Total cell extracts were analyzed by SDS-PAGE, followed by immunoblotting with anti-GDAP1 or anti-Histone $\mathrm{H} 3$ antibodies. Histone $\mathrm{H} 3$ was used as a loading control.

\section{Discussion}

One of the main problems faced by modern medical genetics is the proper identification of a causative mutation followed by determination of the degree of pathogenicity in order to predict the progress of the disease. Furthermore, most genetic disorders are rare, making them very difficult to understand and ultimately to find treatment. It is also extremely expensive to develop efficient treatments for such diseases. These problems also apply to the vast majority of the neurodegenerative heritable disorders. Studies of therapies for neurodegenerative disorders are underdeveloped, even on the preclinical level. In the present study we used, for the first time, a yeast-based model to establish a system in which to assess the pathogenic effect of GDAP1 gene mutations. 
More than 15 years of studies have been devoted to the analysis of GDAP1 mutations. There are some well documented variants such as the GDAP1 $\mathrm{m} 5$ (Leu239Phe) mutation reported in numerous patients. However, several mutations are poorly documented. For example, the GDAP1 m6 (His123Arg) mutation was found only in two families. Thus, testing of pathogenicity is even more necessary in instances such as the GDAP1 $\mathrm{m} 6$ variant $[23,24]$. Even more interesting, and underexplored is the pathogenic status of the GDAP1 $\mathrm{m} 4$ (Glu222Lys) mutation. The GDAP1 m4 mutation has been shown by us to segregate with an extremely mild phenotype in the case of autosomal dominant CMT but in combination with the GDAP1 $\mathrm{m} 5$ (Leu239Phe) mutation it causes severe CMT with autosomal recessive traits [26]. Interestingly, out of the mutations with no clear phenotype in the yeast growth reduction assay, it was the recurrent and pathogenic GDAP1 $\mathrm{m} 5$ mutation that resulted in the highest observed level of mtDNA escape. This may indicate a separate and specific mechanism of action for the GDAP1 m5 mutation. The GDAP1 m8 (Ala156Gly) mutation, which segregates with a CMT with dominant inheritance traits and a moderate clinical pathology, also results in a moderate pathogenic effect in our yeast growth assay. In our study, the GDAP1 m4 (Glu222Lys) mutation has the lowest pathogenic effect (Table 2). Although our previous results showed a clear segregation of the GDAP1 $\mathrm{m} 4$ variant with two CMT phenotypes, its presence has only been documented in two families. There is still a possibility that considering the small group of samples, the association of the GDAP1 $\mathrm{m} 4$ variant with the CMT4A phenotype may be random. Thus, we cannot exclude the possibility that the GDAP1 m4 variant may be categorized as a harmless polymorphism.

Table 2. Pathogenic effect of GDAP1 gene mutations.

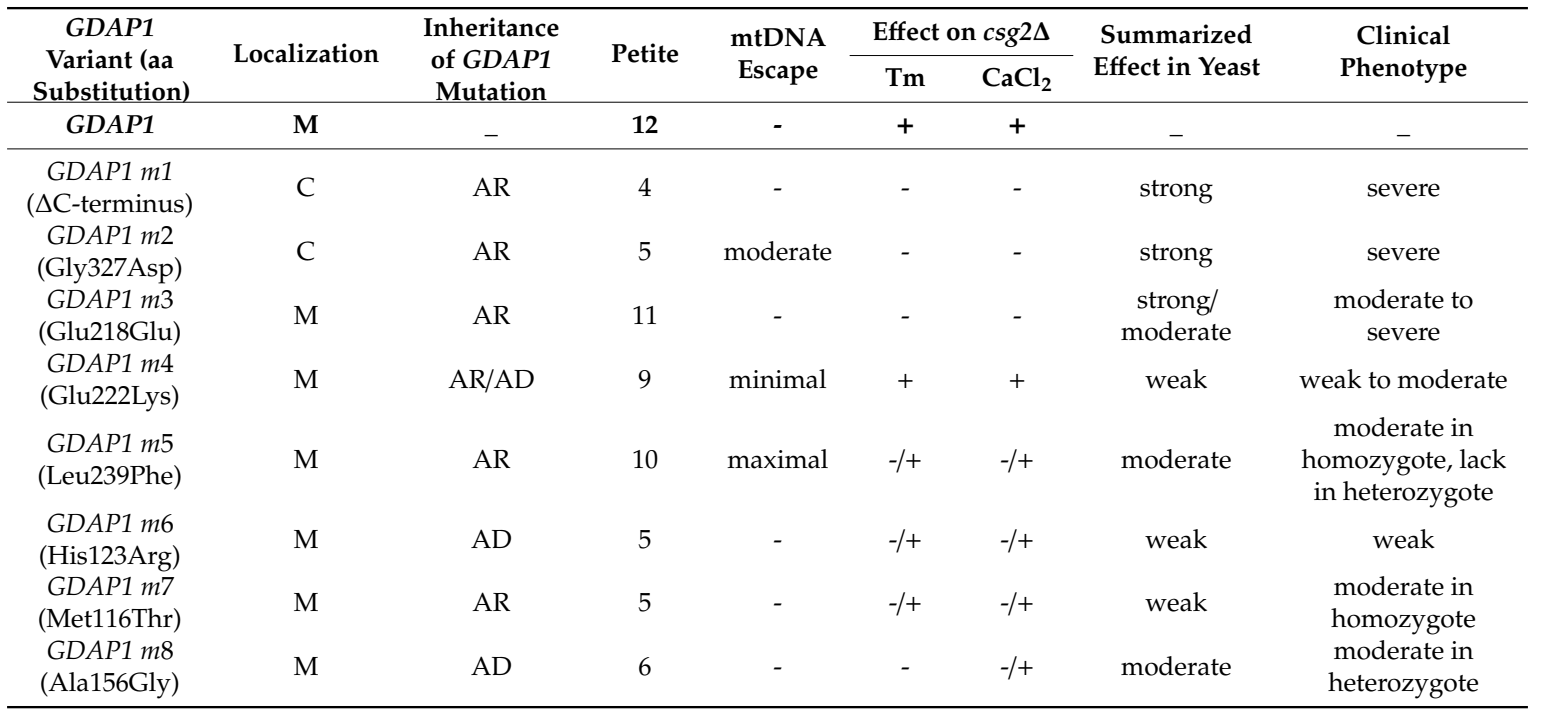

The summarized results illustrating the pathogenic effect of GDAP1 versions analyzed on selected phenotypes in yeast in comparison to the clinical effect. Localization: C-cytoplasmic, M-mitochondrial; Inheritance AR-autosomal recessive, $\mathrm{AD}$ - autosomal dominant; Petite-the results show the percentage of small colonies in the cells expressing GDAP1 variants; $\mathrm{mt}$ DNA escape - the effect of the GDAP1 variants expression on a rate of mitochondrial DNA escape reported as maximal, moderate or minimal; effect of GDAP1 variants expression on the $\operatorname{csg} 2 \Delta$ mutant ability to grow on media containing tunicamycin $(\mathrm{Tm})$ or calcium chloride $\left(\mathrm{CaCl}_{2}\right)(+)$ strong, $(+/-)$ moderate, or $(-)$ no effect.

Finally, our study confirms hypothesis that the GDAP1 mutations located within the transmembrane domain result the severest phenotypes. These mutations lead to the mislocalization of the GDAP1 protein to the cytoplasm instead of to the mitochondrial membrane [25]. In the present study we have shown these types of GDAP1 gene mutations are also associated with the strongest pathogenic effect. This observation is in accordance with previous clinical studies reporting extremely severe phenotypes associated with Glu163X, Ser194X, Thr288fsx290, and Gly327Asp GDAP1 gene mutations. The patients harboring these mutations become wheelchair-dependent during infancy or early adulthood $[8,25]$. We propose that because the localization of the GDAP1 protein to the mitochondrial membrane is critical for its function, the testing of GDAP1 location should be the 
first step in diagnosing the severity of a mutation, especially for mutations affecting the amino acid sequence of the TMD. Furthermore, due to the observed heterogeneity in the mechanisms by which GDAP1 mutations act, the tests used to assess their pathogenicity should be based upon the biological properties/parameters summarized in Table 2.

The model established here is shown to be suitable for validating the pathogenic effect of known GDAP1 mutations. Our system seems to be particularly suited for the validation of new sequence GDAP1 variants identified in single patients manifesting with CMT. The ability to test in a simple yeast-based model is attractive due to its ease of use and low cost. Even when the CMT phenotype has been narrowed by careful clinical and electrophysiological assessment, the whole exome sequencing approach (WES) may generate an average of 2 or 3 variants within CMT genes with ambiguous pathogenic effect [2]. In this instance, access to a reproducible platform for functional studies may be a necessary and useful tool for assessing the causative role of a certain CMT sequence variant.

Author Contributions: Conceptualization, J.K. and A.K.; Methodology, W.R. and J.K.; Validation, W.R., J.K. and A.K.; Formal analysis, J.K.; Investigation, W.R. and J.K.; Resources, W.R., J.K. and D.K.; Data curation, W.R.; Writing-original draft preparation, J.K. and A.K.; Writing-review and editing, W.R., J.K., D.K. and A.K.; Visualization, W.R.; Supervision, A.K.; Project administration, A.K.; Funding acquisition, A.K. All authors have read and agreed to the published version of the manuscript.

Funding: This research was funded by National Science Centre Poland, grant number UMO-2016/23/B/NZ3/02035.

Acknowledgments: The confocal microscopy analysis presented in this study was performed in the Laboratory of Advanced Microscopy Technologies Mossakowski Medical Research Centre, Polish Academy of Sciences. The fluorescence microscopy studies were performed in the Laboratory of Confocal and Fluorescence Microscopy Institute of Biochemistry and Biophysics Polish Academy of Sciences.

Conflicts of Interest: The authors declare no conflict of interest. The funders had no role in the design of the study; in the collection, analyses, or interpretation of data; in the writing of the manuscript, or in the decision to publish the results.

\section{Appendix A}

Table A1. The primer sequences for site directed mutagenesis.

\begin{tabular}{|c|c|c|}
\hline & Primer Name & Primer Sequence \\
\hline \multirow{2}{*}{$\begin{array}{l}\text { GDAP1 m2: c.980G }>A ; \\
\text { p.Gly327Asp }\end{array}$} & GDAP1m2F & $5^{\prime}$-cactcctgcaagcaaatcaaccacaagggtcgt- $3^{\prime}$ \\
\hline & GDAP1m2R & $5^{\prime}$-acgacccttgtggttgatttgcttgcaggagtg- $3^{\prime}$ \\
\hline \multirow{2}{*}{$\begin{array}{l}\text { GDAP1 m3: c. } 652 \mathrm{C}>\mathrm{G} \\
\text { p.Gln218Glu }\end{array}$} & GDAP1m3F & $5^{\prime}$-caattcagtttcaacctcatccaagactttctccaactc-3' \\
\hline & GDAP1m3R & $5^{\prime}$-gagttggagaaagtcttggatgaggttgaaactgaattg- $3^{\prime}$ \\
\hline \multirow{2}{*}{$\begin{array}{c}\text { GDAP1 m4: c.664G >A } \\
\text { p.Glu222Lys }\end{array}$} & GDAP1m4F & $5^{\prime}$-ttcttcatttcttctttgcaatttagtttcaacctgatccaagactt-3' \\
\hline & GDAP1m4R & $5^{\prime}$-agtcttggatcaggttgaaactaaattgcaaagaagaaatgaagaa- $3^{\prime}$ \\
\hline \multirow{2}{*}{$\begin{array}{l}\text { GDAP1 m5: c.715C >T } \\
\text { p.Leu239Phe }\end{array}$} & GDAP1m5F & $5^{\prime}$-ggattcaccgcagaaccaaggttgctggc- $3^{\prime}$ \\
\hline & GDAP1m5R & $5^{\prime}$-gccagcaaccttggttctgcggtgaatcc- $3^{\prime}$ \\
\hline \multirow{2}{*}{$\begin{array}{l}\text { GDAP1 m6: c.368A>G } \\
\text { p.His123Arg }\end{array}$} & GDAP1m6F & $5^{\prime}$-gcagctctcggtaacgttgtacccgtggg- $3^{\prime}$ \\
\hline & GDAP1m6R & $5^{\prime}$-cccacgggtacaacgttaccgagagctgc- $3^{\prime}$ \\
\hline \multirow{2}{*}{$\begin{array}{l}\text { GDAP1 m7: c.347T>C } \\
\text { p.Met116Thr }\end{array}$} & GDAP1m7F & $5^{\prime}$-gtacccgtgggtaatacgtgctttctttatcaggca-3' \\
\hline & GDAP1m7R & $5^{\prime}$-tgcctgataaagaaagcacgtattacccacgggtac- $3^{\prime}$ \\
\hline \multirow{2}{*}{$\begin{array}{l}\text { GDAP1 m8: c.467C >G } \\
\text { p.Ala156Gly }\end{array}$} & GDAP1m8F & $5^{\prime}$-tacgaatccttgtagttccataagccgggatcatg- $3^{\prime}$ \\
\hline & GDAP1m8R & $5^{\prime}$-catgatcccggcttatggaactacaaggattcgta- $3^{\prime}$ \\
\hline
\end{tabular}




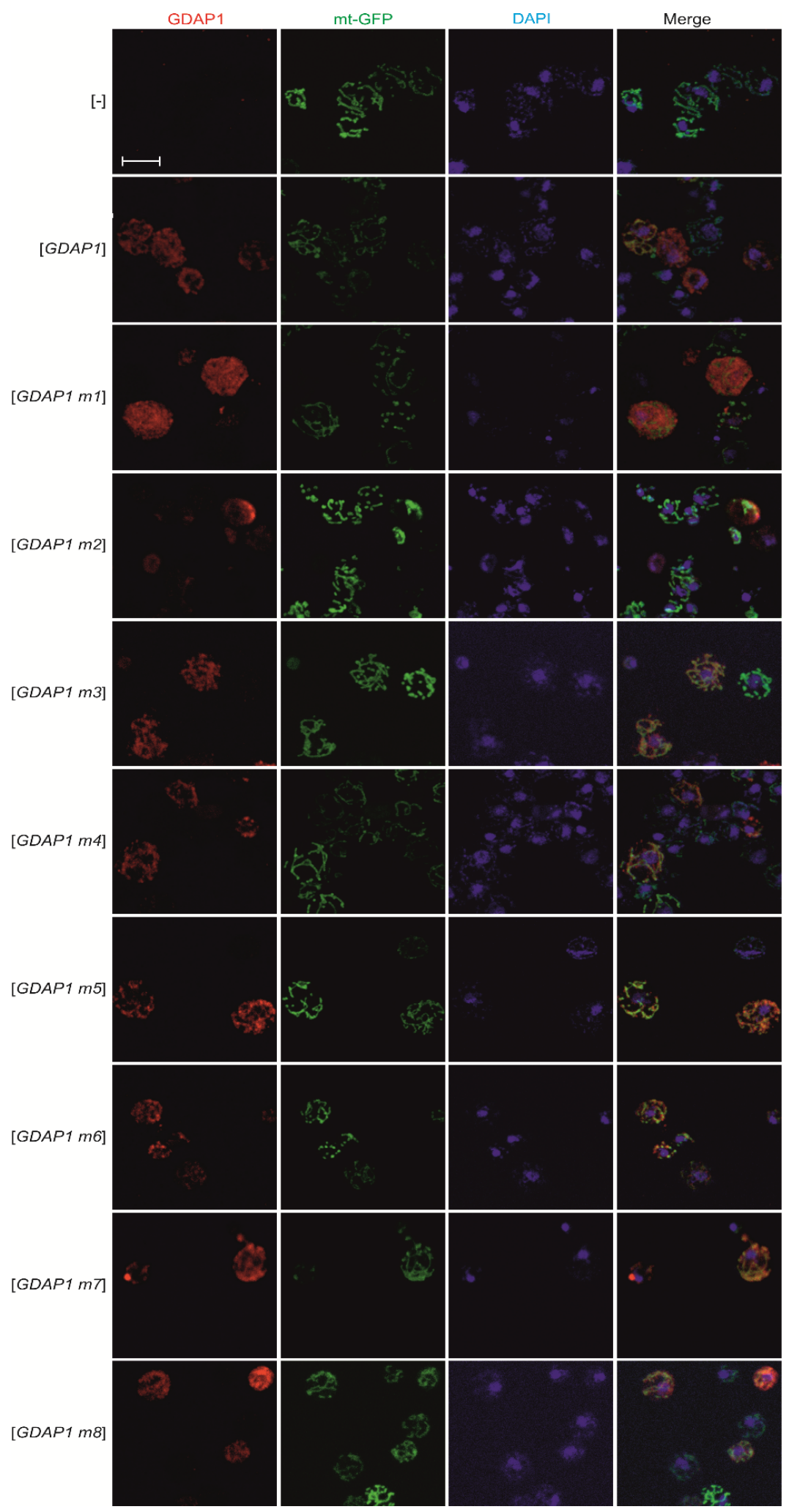

Figure A1. Yeast wild-type cells transformed with plasmid encoding mitochondrially targeted green fluorescent protein (mt-GFP) and with empty vector ([-]) or plasmids encoding GDAP1 alleles (as indicated) were grown in SC-leu-his medium overnight and were then were shifted to glycerol-containing medium for $4 \mathrm{~h}$ to induce expansion of mitochondria. Cells were fixed, stained, and observed using confocal microscopy. GDAP1 was visualized by immunostaining (red), mitochondria using the mt-GFP protein marker (green) and the nucleus was stained with DAPI (blue). Scale bar $5 \mu \mathrm{m}$. 


\section{References}

1. Lupski, J.R.; Belmont, J.W.; Boerwinkle, E.; Gibbs, R.A. Clan Genomics and the Complex Architecture of Human Disease. Cell 2011, 147, 32-43. [CrossRef]

2. Gonzaga-Jauregui, C.; Harel, T.; Gambin, T.; Kousi, M.; Griffin, L.B.; Francescatto, L.; Ozes, B.; Karaca, E.; Jhangiani, S.N.; Bainbridge, M.N.; et al. Exome Sequence Analysis Suggests that Genetic Burden Contributes to Phenotypic Variability and Complex Neuropathy. Cell Rep. 2015, 12, 1169-1183. [CrossRef]

3. Baxter, R.V.; Ben Othmane, K.; Rochelle, J.M.; Stajich, J.E.; Hulette, C.; Dew-Knight, S.; Hentati, F.; Ben Hamida, M.; Bel, S.; Stenger, J.E.; et al. Ganglioside-induced differentiation-associated protein-1 is mutant in Charcot-Marie-Tooth disease type 4A/8q21. Nat. Genet. 2002, 30, 21-22. [CrossRef]

4. Cuesta, A.; Pedrola, L.; Sevilla, T.; García-Planells, J.; Chumillas, M.J.; Mayordomo, F.; LeGuern, E.; Marín, I.; Vílchez, J.J.; Palau, F. The gene encoding ganglioside-induced differentiation-associated protein 1 is mutated in axonal Charcot-Marie-Tooth type 4A disease. Nat. Genet. 2002, 30, 22-25. [CrossRef]

5. González-Sánchez, P.; Satrústegui, J.; Palau, F.; del Arco, A. Calcium Deregulation and Mitochondrial Bioenergetics in GDAP1-Related CMT Disease. Int. J. Mol. Sci. 2019, 20, 403. [CrossRef] [PubMed]

6. Rzepnikowska, W.; Kochański, A. A role for the GDAP1 gene in the molecular pathogenesis of Charcot-Marie-Tooth disease. Acta Neurobiol. Exp. (Wars) 2018, 78, 1-13. [CrossRef] [PubMed]

7. Azzedine, H.; Ruberg, M.; Ente, D.; Gilardeau, C.; Périé, S.; Wechsler, B.; Brice, A.; LeGuern, E.; Dubourg, O. Variability of disease progression in a family with autosomal recessive CMT associated with a S194X and new R310Q mutation in the GDAP1 gene. Neuromuscul. Disord. 2003, 13, 341-346. [CrossRef]

8. Claramunt, R.; Pedrola, L.; Sevilla, T.; López de Munain, A.; Berciano, J.; Cuesta, A.; Sánchez-Navarro, B.; Millán, J.M.; Saifi, G.M.; Lupski, J.R.; et al. Genetics of Charcot-Marie-Tooth disease type 4A: Mutations, inheritance, phenotypic variability, and founder effect. J. Med. Genet. 2005, 42, 358-365. [CrossRef]

9. Liu, H.; Nakagawa, T.; Kanematsu, T.; Uchida, T.; Tsuji, S. Isolation of 10 differentially expressed cDNAs in differentiated Neuro2a cells induced through controlled expression of the GD3 synthase gene. J. Neurochem. 1999, 72, 1781-1790. [CrossRef]

10. Wagner, K.M.; Rüegg, M.; Niemann, A.; Suter, U. Targeting and Function of the Mitochondrial Fission Factor GDAP1 Are Dependent on Its Tail-Anchor. PLoS ONE 2009, 4, e5160. [CrossRef]

11. Pedrola, L.; Espert, A.; Wu, X.; Claramunt, R.; Shy, M.E.; Palau, F. GDAP1, the protein causing Charcot-Marie-Tooth disease type 4A, is expressed in neurons and is associated with mitochondria. Hum. Mol. Genet. 2005, 14, 1087-1094. [CrossRef] [PubMed]

12. Pedrola, L.; Espert, A.; Valdés-Sánchez, T.; Sánchez-Piris, M.; Sirkowski, E.E.; Scherer, S.S.; Fariñas, I.; Palau, F. Cell expression of GDAP1 in the nervous system and pathogenesis of Charcot-Marie-Tooth type 4A disease. J. Cell. Mol. Med. 2008, 12, 679-689. [CrossRef] [PubMed]

13. Niemann, A.; Ruegg, M.; La Padula, V.; Schenone, A.; Suter, U. Ganglioside-induced differentiation associated protein 1 is a regulator of the mitochondrial network. J. Cell Biol. 2005, 170, 1067-1078. [CrossRef] [PubMed]

14. Niemann, A.; Wagner, K.M.; Ruegg, M.; Suter, U. GDAP1 mutations differ in their effects on mitochondrial dynamics and apoptosis depending on the mode of inheritance. Neurobiol. Dis. 2009, 36, 509-520. [CrossRef] [PubMed]

15. González-Sánchez, P.; Pla-Martín, D.; Martínez-Valero, P.; Rueda, C.B.; Calpena, E.; Del Arco, A.; Palau, F.; Satrústegui, J. CMT-linked loss-of-function mutations in GDAP1 impair store-operated Ca(2+) entry-stimulated respiration. Sci. Rep. 2017, 7, 42993. [CrossRef] [PubMed]

16. Pla-Martín, D.; Rueda, C.B.; Estela, A.; Sánchez-Piris, M.; González-Sánchez, P.; Traba, J.; de la Fuente, S.; Scorrano, L.; Renau-Piqueras, J.; Alvarez, J.; et al. Silencing of the Charcot-Marie-Tooth disease-associated gene GDAP1 induces abnormal mitochondrial distribution and affects $\mathrm{Ca}^{2+}$ homeostasis by reducing store-operated $\mathrm{Ca}^{2+}$ entry. Neurobiol. Dis. 2013, 55, 140-151. [CrossRef]

17. Niemann, A.; Huber, N.; Wagner, K.M.; Somandin, C.; Horn, M.; Lebrun-Julien, F.; Angst, B.; Pereira, J.A.; Halfter, H.; Welzl, H.; et al. The Gdap1 knockout mouse mechanistically links redox control to Charcot-Marie-Tooth disease. Brain 2014, 137, 668-682. [CrossRef]

18. Noack, R.; Frede, S.; Albrecht, P.; Henke, N.; Pfeiffer, A.; Knoll, K.; Dehmel, T.; Meyer zu Horste, G.; Stettner, M.; Kieseier, B.C.; et al. Charcot-Marie-Tooth disease CMT4A: GDAP1 increases cellular glutathione and the mitochondrial membrane potential. Hum. Mol. Genet. 2012, 21, 150-162. [CrossRef] 
19. Estela, A.; Pla-Martín, D.; Sánchez-Piris, M.; Sesaki, H.; Palau, F. Charcot-Marie-Tooth-related gene GDAP1 complements cell cycle delay at G2/M phase in Saccharomyces cerevisiae fis1 gene-defective cells. J. Biol. Chem. 2011, 286, 36777-36786. [CrossRef]

20. Thorsness, P.E.; Fox, T.D. Nuclear mutations in Saccharomyces cerevisiae that affect the escape of DNA from mitochondria to the nucleus. Genetics 1993, 134, 21-28.

21. Mumberg, D.; Müller, R.; Funk, M. Yeast vectors for the controlled expression of heterologous proteins in different genetic backgrounds. Gene 1995, 156, 119-122. [CrossRef]

22. Westermann, B.; Neupert, W. Mitochondria-targeted green fluorescent proteins: Convenient tools for the study of organelle biogenesis in Saccharomyces cerevisiae. Yeast 2000, 16, 1421-1427. [CrossRef]

23. Zimon, M.; Baets, J.; Fabrizi, G.M.; Jaakkola, E.; Kabzinska, D.; Pilch, J.; Schindler, A.B.; Cornblath, D.R.; Fischbeck, K.H.; Auer-Grumbach, M.; et al. Dominant GDAP1 mutations cause predominantly mild CMT phenotypes. Neurology 2011, 77, 540-548. [CrossRef] [PubMed]

24. Kabzińska, D.; Strugalska-Cynowska, H.; Kostera-Pruszczyk, A.; Ryniewicz, B.; Posmyk, R.; Midro, A.; Seeman, P.; Báranková, L.; Zimoń, M.; Baets, J.; et al. L239F founder mutation in GDAP1 is associated with a mild Charcot-Marie-Tooth type 4C4 (CMT4C4) phenotype. Neurogenetics 2010, 11, 357-366. [CrossRef]

25. Kabzińska, D.; Niemann, A.; Drac, H.; Huber, N.; Potulska-Chromik, A.; Hausmanowa-Petrusewicz, I.; Suter, U.; Kochański, A. A new missense GDAP1 mutation disturbing targeting to the mitochondrial membrane causes a severe form of AR-CMT2C disease. Neurogenetics 2011, 12, 145-153. [CrossRef]

26. Kabzińska, D.; Kotruchow, K.; Cegielska, J.; Hausmanowa-Petrusewicz, I.; Kochański, A. A severe recessive and a mild dominant form of Charcot-Marie-Tooth disease associated with a newly identified Glu222Lys GDAP1 gene mutation. Acta Biochim. Pol. 2014, 61, 739-744. [CrossRef]

27. Lewis, S.C.; Uchiyama, L.F.; Nunnari, J. ER-mitochondria contacts couple mtDNA synthesis with mitochondrial division in human cells. Science 2016, 353, aaf5549. [CrossRef]

28. Murley, A.; Lackner, L.L.; Osman, C.; West, M.; Voeltz, G.K.; Walter, P.; Nunnari, J. ER-associated mitochondrial division links the distribution of mitochondria and mitochondrial DNA in yeast. eLife 2013, 2, e00422. [CrossRef]

29. Campbell, C.L.; Thorsness, P.E. Escape of mitochondrial DNA to the nucleus in yme1 yeast is mediated by vacuolar-dependent turnover of abnormal mitochondrial compartments. J. Cell Sci. 1998, 111 Pt 1, 2455-2464.

30. Thorsness, M.K.; White, K.H.; Thorsness, P.E. Migration of mtDNA into the Nucleus. In Mitochondrial DNA.; Humana Press: New Jersey, NJ, USA, 2002; pp. 177-186.

31. Shafer, K.S.; Hanekamp, T.; White, K.H.; Thorsness, P.E. Mechanisms of mitochondrial DNA escape to the nucleus in the yeast Saccharomyces cerevisiae. Curr. Genet. 1999, 36, 183-194. [CrossRef]

32. Xu, N.; Thorsness, M.K.; Thorsness, P.E. Mitochondrial DNA impacts the morphology of mitochondrial compartments. Gene 2005, 354, 37-42. [CrossRef] [PubMed]

33. Knupp, J.; Martinez-Montañés, F.; Van Den Bergh, F.; Cottier, S.; Schneiter, R.; Beard, D.; Chang, A. Sphingolipid accumulation causes mitochondrial dysregulation and cell death. Cell Death Differ. 2017, 24, 2044-2053. [CrossRef] [PubMed]

(C) 2020 by the authors. Licensee MDPI, Basel, Switzerland. This article is an open access article distributed under the terms and conditions of the Creative Commons Attribution (CC BY) license (http://creativecommons.org/licenses/by/4.0/). 